\title{
Cooperatively Breeding Cottontop Tamarins (Saguinus oedipus) Do Not Donate Rewards to Their Long-Term Mates
}

\author{
Katherine A. Cronin, Kori K. E. Schroeder, \\ and Emily S. Rothwell \\ University of Wisconsin-Madison
}

\author{
Joan B. Silk \\ University of California at Los Angeles
}

Charles T. Snowdon

University of Wisconsin-Madison

\begin{abstract}
This study tested the hypothesis that cooperative breeding facilitates the emergence of prosocial behavior by presenting cottontop tamarins (Saguinus oedipus) with the option to provide food rewards to pair-bonded mates. In Experiment 1, tamarins could provide rewards to mates at no additional cost while obtaining rewards for themselves. Contrary to the hypothesis, tamarins did not demonstrate a preference to donate rewards, behaving similar to chimpanzees in previous studies. In Experiment 2, the authors eliminated rewards for the donor for a stricter test of prosocial behavior, while reducing separation distress and food preoccupation. Again, the authors found no evidence for a donation preference. Furthermore, tamarins were significantly less likely to deliver rewards to mates when the mate displayed interest in the reward. The results of this study contrast with those recently reported for cooperatively breeding common marmosets, and indicate that prosocial preferences in a food donation task do not emerge in all cooperative breeders. In previous studies, cottontop tamarins have cooperated and reciprocated to obtain food rewards; the current findings sharpen understanding of the boundaries of cottontop tamarins' food-provisioning behavior.
\end{abstract}

Keywords: donation, prosocial behavior, cooperative breeding, cottontop tamarin

Supplemental materials: http://dx.doi.org/10.1037/a0015094.supp

Many recent attempts have been made to identify evolutionary origins of the human tendency to assist others, or to act prosocially. These searches began reasonably with the closest living relative of humans, chimpanzees (Pan troglodytes). However, when chimpanzees have been presented with food donation tasks that allowed them to provide rewards to conspecifics at little or no cost to themselves, they have not shown a preference to do so (Jensen, Hare, Call, \& Tomasello, 2006; Silk et al., 2005; Vonk et al., 2008). The lack of evidence for prosocial behavior in chimpanzees has led to the consideration of two quite different hypotheses. One hypothesis is that the human tendency to act in the best interest of others is a derived trait that originated after the divergence of the human and chimpanzee lineages. A second, broader

Katherine A. Cronin, Kori K. E. Schroeder, Emily S. Rothwell, and Charles T. Snowdon, Department of Psychology, University of WisconsinMadison; Joan B. Silk, Department of Anthropology, University of California, Los Angeles.

We thank Andrew Mulder for construction of the apparatus, Bob Becker for creation of figures, Daniel Acheson for statistical advice, and the management staff of the Callitrichid laboratory. This research was supported by National Institute of Mental Health Grant MH029775, the University of Wisconsin Graduate School Research Committee, a Hilldale Professorship to Charles T. Snowdon, and a National Science Foundation Graduate Research Fellowship to Katherine A. Cronin.

Correspondence concerning this article should be addressed to Katherine A. Cronin, Department of Psychology, University of Wisconsin, 1202 West Johnson Street, Madison, WI 53706. E-mail: kacronin@wisc.edu hypothesis posits that the tendency to provide benefits to conspecifics is not uniquely human but a result of some combination of social or ecological selective pressures.

Although chimpanzees are closely related to humans, they are socially distinct. Chimpanzees are characterized by fission-fusion social organization, and communities frequently fragment into smaller temporary parties (reviewed in Muller \& Mitani, 2005). Males remain in their natal communities throughout their lives, whereas females typically disperse when they reach sexual maturity. Males are more sociable than females, and social bonds among males are generally stronger than bonds among females (Muller \& Mitani, 2005). Males participate in a wide range of cooperative activities, including grooming, mate-guarding coalitions (e.g., Watts, 1998), coalitionary aggression (e.g., Nishida, 1983; Riss \& Goodall, 1977), hunting (e.g., Boesch, 2002), and boundary patrols (Watts \& Mitani, 2001). Although males frequently cooperate, they also compete fiercely for high-ranking positions within their groups, and high-ranking males monopolize access to resources, including receptive females.

The competitive social environment of chimpanzees has been reflected in some laboratory studies of chimpanzee cognition. Hare and Tomasello (2004) reported that chimpanzees were more skillful at tasks administered in competitive rather than cooperative contexts. However, congeneric bonobos (Pan paniscus), which are more socially tolerant and less aggressive than chimpanzees, were more successful than chimpanzees at cooperating to obtain a monopolizable food reward (Hare, Melis, Woods, Hastings, \& 
Wrangham, 2007). Therefore, although phylogenetic similarity is often a primary consideration in comparative psychology, social factors that might influence the emergence of a trait must be considered, and the possibility for convergent evolution between more distantly related species should also be explored.

The cooperatively breeding Callithrichidae (marmosets and tamarins) share many social characteristics with humans. Callithrichids, like many humans, exhibit long-lasting heterosexual pair bonds, and offspring are cared for by both parents as well as by older siblings and occasionally unrelated group members. Members of Callithrichid social groups actively share food with and carry infants (Feistner \& McGrew, 1989; Snowdon, 1996). Behavioral coordination among group members is well developed, with multiple individuals collaborating and exchanging roles to provide care for infants, locate food, watch for predators, and defend their territory. The number of available helpers is positively correlated with offspring survival in both field and laboratory environments (Savage, Giraldo, Soto, \& Snowdon, 1996; Snowdon, 1996). In laboratory tasks, unrelated pair-bonded cottontop tamarins (Saguinus oedipus) cooperated to obtain food rewards, and continued to do so even when only one individual benefited from the cooperative act (Cronin, Kurian, \& Snowdon, 2005; Cronin \& Snowdon, 2008). Hauser, Chen, Chen, and Chuang (2003) demonstrated that cottontop tamarins discriminated between reciprocating and nonreciprocating individuals and differentiated between selfish and altruistic actions of conspecifics. Consideration of the social characteristics of cooperative breeders has led to a specific hypothesis that prosocial behavior has evolved in cooperative breeding species (Burkart, Fehr, Efferson, \& van Schaik, 2007).

It is becoming evident that cooperative breeding is not the only social environment that may facilitate prosocial behavior on some food donation tasks. In recent studies of prosocial preferences in capuchin monkeys (Cebus apella), a species characterized by high social tolerance but not cooperative breeding, capuchins did provide rewards to conspecifics when given the option to do so while obtaining rewards for themselves (de Waal, Leimgruber, \& Greenberg, 2008; Lakshminarayanan \& Santos, 2008). However, we do not yet know whether capuchins would demonstrate prosocial behavior when the donor does not receive any rewards for its actions. To our knowledge, this stricter version of prosocial preferences has not yet been tested in capuchin monkeys.

We hypothesized that the cooperative social organization of Callithrichids would have favored selection for prosocial behavior and tested that hypothesis here with cottontop tamarins. In an independent study, Burkart et al. (2007) arrived at the same hypothesis and presented cooperatively breeding common marmosets (Callithrix jacchus) with a food donation task very similar to that used with chimpanzees in previous studies. Burkart and colleagues found that common marmosets, unlike chimpanzees, did provide food to conspecifics, even when donors were unable to obtain rewards for themselves. This finding supports the hypothesis that prosocial behaviors may emerge in cooperatively breeding species. However we report here that cottontop tamarins performed differently on a nearly identical task, indicating that influences other than cooperative breeding must be contributing to the emergence of prosocial behavior.

\section{Experiment 1: Donation as a Byproduct of Selfish Action}

In the first experiment, tamarins were presented with the choice between two trays, one that delivered a single reward to themselves and a single reward to their mate (the " $1 / 1$ " option), and a second that delivered a single reward to themselves only (the " $1 / 0$ " option). Tamarins were presented with mate-present and mateabsent (control) conditions. The methodology was closely modeled after the study of chimpanzee prosociality by Silk et al. (2005), with slight adaptations to allow testing of cottontop tamarins. Prior to inclusion in the study, the tamarins participated in a series of training stages with strict criteria to ensure task understanding.

\section{Method}

\section{Subjects}

The subjects of this study were eight adult male-female pairs of cottontop tamarins (Saguinus oedipus). Each pair had been housed together for at least 1 year prior to the onset of training (range $=$ 1.0 to 9.4 years, median $=5.5$ years), and had no offspring present. They ranged in age from 2.5 to 11.5 years (median $=7.0$ years). Two pairs (Sc and In, Ga and $\mathrm{He}$ ) had been subjects of previous cooperative problem-solving experiments (Cronin et al., 2005; Cronin \& Snowdon, 2008).

\section{Apparatus}

Overview. The experimental setup consisted of a recipient cage that hung inside the tamarins' home cage and separated the donor and recipient from each other. The donation apparatus hung on the outside of the home cage and spanned the donor and recipient locations. Only the donor had access to the tray handles. An auxiliary cage connected to the home cage was used during mate-absent sessions (see Figure 1).

Home cage. The tamarins were housed in indoor cages constructed of anodized aluminum framing and polyurethane-coated steel mesh. Cages measured $236 \times 160 \times 93 \mathrm{~cm}$ (length $\times$ width $\times$ height). Cages included natural branches, wooden planks, and ropes to simulate an arboreal environment. All tamarins were housed in rooms containing multiple cages. Cages were isolated from each other visually by opaque fabric sheets. For additional husbandry information, see Ginther, Ziegler, and Snowdon (2001).

Donation apparatus. The donation apparatus consisted of two clear polycarbonate trays in a stainless steel frame. The trays were on a pulley system so when a tamarin pulled one tray toward itself, the second tray moved in the opposite direction and remained out of reach until manually reset by the experimenter. The trays were $31 \mathrm{~cm}$ wide and $5 \mathrm{~cm}$ deep and the vertical distance between the two trays was $5 \mathrm{~cm}$. Each tray had two reward locations positioned $23 \mathrm{~cm}$ apart. Hooks on the apparatus allowed the apparatus to be fastened to the outside of the home cage. A metal handle extended from one side of each tray to within reach of the donor tamarin ranging in the home cage; the distance between the handle and the front of the tamarins' home cage was $8 \mathrm{~cm}$. The apparatus was positioned so the reward locations on one side of the tray were in front of the donor and the reward locations on the other side of the 


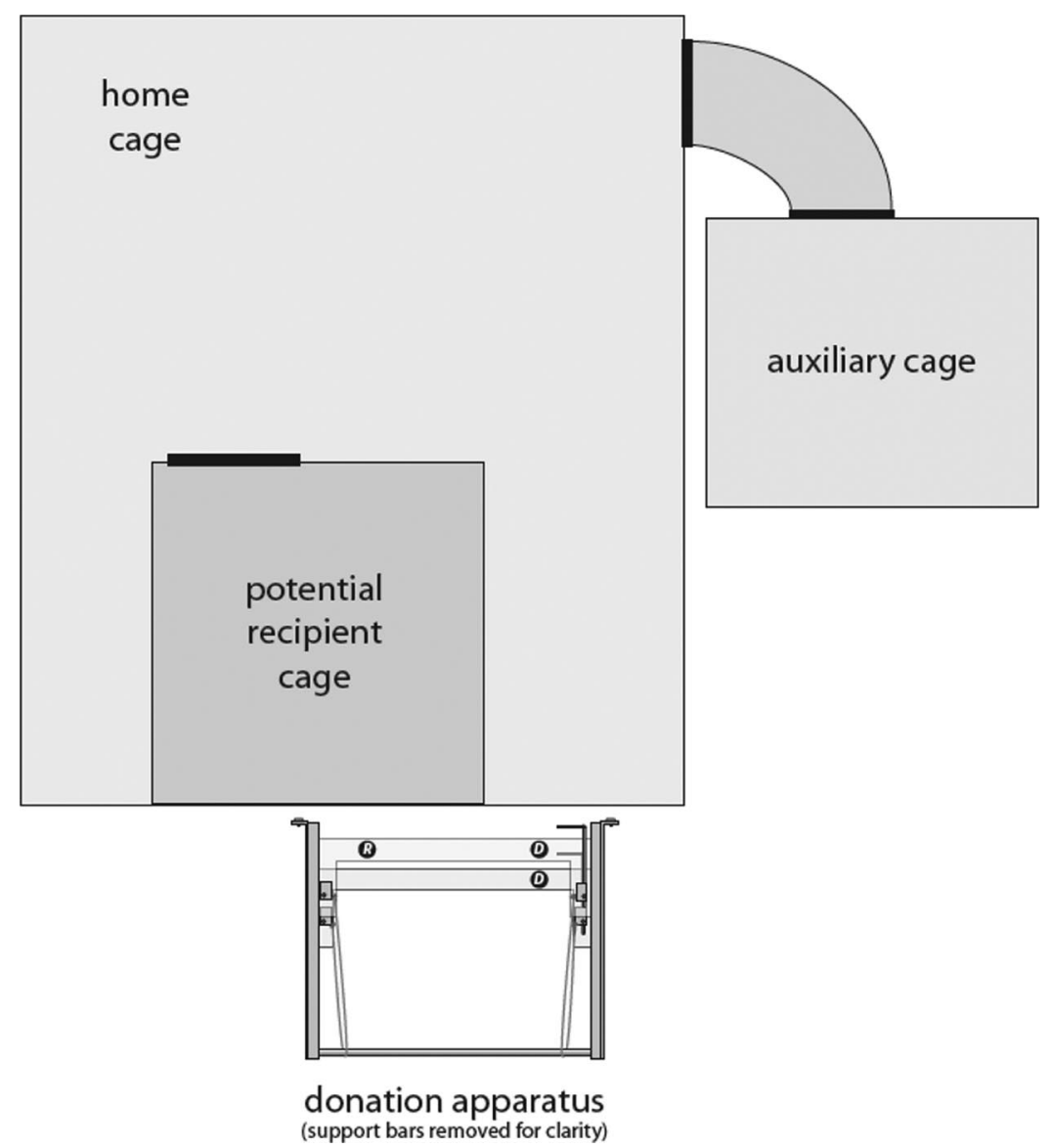

Figure 1. Top-down view of the setup for Experiment 1. During mate-present conditions, the donor ranged in the home cage and had access to the handles on the donation apparatus, and the potential recipient was restricted to the recipient cage within the home cage. During mate-absent (control) conditions, the donor ranged in the home cage and the mate was restricted to the auxiliary cage outside the home cage. The donation apparatus hung on the outside of the home cage. Each circle on the trays of the donation apparatus represents a reward, labeled "D" for rewards accessible by the donor and "R" for the reward accessible by the recipient. In this example, the top tray would be the "1/1" tray (provides one reward to the donor and one reward to the recipient) and the bottom tray would be the " $1 / 0$ " tray (provides one reward to the donor and no rewards to the recipient).

tray were in front of the recipient (or empty recipient cage, depending on condition; see supplemental photograph 1).

Potential recipient cage (hereafter referred to as recipient cage). The recipient cage was constructed of anodized aluminum framing and mesh faces, and measured $51 \times 46 \times 61 \mathrm{~cm}$ (length $\times$ width $\times$ height). The openings in the mesh were $1.25 \times$ $2.5 \mathrm{~cm}$. The recipient cage was constructed of the same materials as the tamarins' home cages. The recipient cage had two steel hooks and hung on the inside of the home cage door. Unlike all other faces of the recipient cage, the face of the recipient cage that was flush with the home cage door was not covered in mesh, allowing the mesh of the door to the home cage to serve as the front wall of the recipient cage. A piece of mesh was removed from the rear wall of the recipient cage; this hole was covered with an opaque nonflexible plastic door. This door was opened and closed by the experimenter from outside of the home cage using wire (see supplemental photograph 2).

Auxiliary cage. An auxiliary cage measuring $66 \times 64 \times 188$ cm (length $\times$ width $\times$ height) was located outside the home cage and connected to the home cage with $15.2 \mathrm{~cm}$ diameter ventilation ducting made of thermoplastic urethane with internal wires. The distance between the auxiliary cage and the home cage varied across pairs because of spatial restrictions within each room and ranged between 30 and $90 \mathrm{~cm}$. The auxiliary cage was constructed of the same materials as the home cage and recipient cage. Both ends of the tubing were covered by an opaque nonflexible plastic door that could be opened and closed by the experimenter using wire.

\section{Training Procedure}

The tamarins were habituated to the recipient cage and trained to operate the donation apparatus through shaping and positive reinforcement. The training program was designed so the tamarins would never be reinforced for choosing a tray with two rewards to eliminate any possibility that the tamarins would be inadvertently trained to choose the two-reward tray over the one-reward tray. Therefore, all training used trays with single rewards only. 
Stage 1. The tamarins were habituated to the recipient cage by incrementally increasing the amount of time they were isolated in the recipient cage and providing positive reinforcement (small food rewards at increasing intervals) until tamarins consistently demonstrated lack of distress in the recipient cage for at least 8 consecutive min. The number of sessions required to achieve this level of habituation ranged from 1 to 35 sessions (median $=15.5$ sessions). All tamarins completed Stage 1 before testing; however, some tamarins began Stage 2 training prior to completing Stage 1.

Stage 2. During initial training sessions with the donation apparatus, one tray was removed from the apparatus. A single reward was placed on the remaining tray near the handle. Both tamarins were present in the home cage for Stage 2. The recipient cage was not present. When a tamarin placed its hand on the handle, the experimenter would slide the tray toward the tamarin, allowing the food to come into reach. In addition, small rewards were sometimes placed on the handle or near the apparatus to increase interest and likelihood of reaching for the handle. A tamarin completed this training stage when it fully pulled the tray and retrieved the reward without any experimenter involvement on $90 \%$ of trials $(n=10)$ over 2 consecutive sessions. The number of sessions required to achieve this criterion ranged from 2 to 34 sessions (median $=5.5$ sessions). Tamarins proceeded to Stage 3 once both individuals in the pair had completed Stage 2.

Stage 3. The second tray was inserted into the apparatus, and the apparatus was baited so that there was a single reward in one of four locations (donor side-top tray, donor side-bottom tray, recipient side-top tray, recipient side_-bottom tray). The baited location was chosen on the basis of a predetermined, randomized, counterbalanced design. The recipient cage was not present in the cage. A tamarin completed this training stage when it chose the baited tray and retrieved the reward on 17 of 20 pulls over two consecutive sessions, with no single session performance lower than 8 of 10. The criteria chosen for this stage would be difficult to meet by chance; the probability of making 17 of 20 correct choices is .002 (two-tailed binomial test). The number of sessions required to achieve this criterion ranged from 2 to 15 sessions (median $=8$ sessions). Tamarins proceeded to Stage 4 once both individuals in the pair had completed Stage 3 .

Stage 4. Each tamarin was tested alone in the home cage with the donation apparatus and the recipient cage present while their partner was temporarily isolated in the auxiliary cage. The door to the recipient cage was secured in the open position so the tamarin could freely enter and exit. Both trays were present in the donation apparatus, and a single reward was randomly placed in one of the four locations as before. To successfully solve a trial, the tamarin was required to choose the baited tray and retrieve the reward from the correct location within $30 \mathrm{~s}$ (on half the trials this required the tamarin to enter the recipient cage) on 17 of 20 trials in 2 consecutive sessions, with no single session performance lower than 8 of 10. Again, the probability of making 17 of 20 correct choices is .002 (two-tailed binomial test). The number of sessions required to achieve this criterion ranged from 3 to 16 sessions (median $=7$ sessions). Two male tamarins did not pass Stage 4 because of an inability to focus on the apparatus when their mate was in the auxiliary cage; therefore, they were not included as donors but were included as recipients. Because the tamarins had to choose the correct location and travel to the location to which the reward was delivered to proceed to testing, we could be reasonably con- fident that the tamarins were aware of the position to which rewards would be delivered when selecting a tray.

\section{Testing Procedure}

Testing commenced once training criteria had been met. Each potential donor (hereafter referred to as donor) participated in four sessions, once daily for 4 consecutive days. All sessions took place between 9:30 and 11:30 a.m. or 2:30 and 4:30 p.m. The time of testing (a.m. or p.m.) was held constant for each pair. Sessions were alternated between partner-present and partner-absent (control) conditions, and the first condition and sex of the first individual to serve as donor were randomly assigned and counterbalanced across individuals

Sessions consisted of 20 trials. On each trial, one tray was baited with two rewards and one with a single reward; therefore, if the double-baited tray was selected, a single reward would be in front of the donor and a single reward in front of the recipient (the " $1 / 1$ " tray), and the other tray provided a reward for the donor only (the " $1 / 0$ " tray). Therefore, on every trial the donor was assured a reward, but only one of the two trays would allow the recipient a reward. This reward scheme is the same as that employed by Silk et al. (2005) in the study of chimpanzee prosociality.

Once a donor completed four sessions, the roles were reversed and the former potential recipient (hereafter referred to as recipient) served as donor for the next four sessions (except for the two subjects whose mates did not meet the training criteria described above). Two to 3 days elapsed between role reversals.

During the mate-present condition, the donor ranged in the home cage and the recipient was restricted to the recipient cage. When the donor and recipient were positioned in front of the donation apparatus, a single piece of mesh (with $1.25 \mathrm{~cm} \times 2.5 \mathrm{~cm}$ openings) separated, them allowing visual, olfactory, auditory, and tactile contact.

During the mate-absent (control) condition, the donor ranged in the home cage and the mate was isolated in the auxiliary cage outside the home cage. The recipient cage was in the same location as the partner-present condition, and was secured shut (see Figure 1).

Rewards were identified during training sessions that motivated both individuals within a pair. This determined the single reward type used for the pair throughout testing (cookie, raisin, tuna, peanut, or burger). The location of the $1 / 1$ tray was randomized between the top and bottom locations, and the randomization was restricted so that within each session the 1/1 tray was in the top position on 10 trials and in the bottom position on 10 trials. All rewards were of equal size. A trial began when the experimenter (KAC) pushed the tray handles within reach of the donor. The experimenter did not push the tray handles into reach until the donor oriented his or her head toward the reward located in front of the recipient to increase the likelihood that the donor attended to the reward locations. The trial ended when the donor pulled a handle to bring one of the trays into reach of the donor and recipient. Subject responses (tray choice) were coded on a data sheet during the session by KAC.

\section{Data Analysis}

The data were analyzed at both the group and individual levels. Analyses were performed using SPSS version 12.0 and the online 
statistical package VassarStats (Lowry, 2008). Results were considered significant at $p \leq .05$.

Group analyses. To determine whether tamarins were more likely to choose the $1 / 1$ tray when their partner was present compared with when their partner was absent, we performed a binomial logistic regression. Tray choice (1/1 or $1 / 0)$ was the dependent variable, and condition (present or absent), block (first or second round of conditions), and position (1/1 tray on top or bottom) were covariates. Because the data included repeated measures from the same individuals, we included individual identity as a categorical variable to control for individual differences and remove the source of nonindependence.

Individual analyses. To determine whether any individual tamarin was more likely to choose the $1 / 1$ tray when the mate was present compared with when the mate was absent, we performed a two-tailed Fisher's exact test on the data for each tamarin, collapsing across blocks.

\section{Results}

\section{Group Results}

Donor tamarins made a response and retrieved the reward from their selected tray on every trial. Tamarins chose the $1 / 1$ tray an average of $56.0 \%$ (95\% confidence interval $[\mathrm{CI}]=50.7,61.4)$ of trials during the mate-present condition and $52.5 \%(95 \% \mathrm{CI}=$ $48.5,56.5)$ of trials during the mate-absent condition. The results of the binomial logistical regression failed to support our hypothesis. Had the tamarins chosen the 1/1 tray more often when their partner was present than when their partner was absent, we should have found a significant effect of condition. However, condition was nonsignificant (odds ratio $[\mathrm{OR}]=0.870, p=.250$ ), as were tray position $(\mathrm{OR}=1.166, p=.204)$ and block $(\mathrm{OR}=1.117, p=$ .362 ). In addition, there was no sex difference observed (two-tailed independent samples $t$ test on difference scores between proportion of $1 / 1$ choices present vs. absent), $t(12)=0.540, p=.599, d=$ 0.293 .

\section{Individual Results}

Individual analyses were nonsignificant for 13 of 14 individuals (all $p \mathrm{~s} \geq .11$ ). The Fisher's exact test for 1 individual (a female,
Wi) resulted in significance ( $p=.041$ ); however, the direction was in the opposite of that predicted, that is, the female chose the $1 / 1$ option more often when the partner was absent than when the partner was present (see Figure 2).

\section{Discussion}

We found no evidence that tamarins pulled the double-baited tray more often when a recipient was present, as was the case for the Silk et al. (2005) study with chimpanzees using a similar experimental design. It is possible that a preoccupation with food rewards may have overridden the tamarins' ability to donate in this experiment. Food preoccupation has been hypothesized to inhibit chimpanzees' ability to perform prosocial actions, and in some situations that do not involve food, chimpanzees have behaved in the interest of others (Hirata, 2007; Warneken, Hare, Melis, Hanus, $\&$ Tomasello, 2007). The presence of food rewards has been shown to inhibit demonstration of some cognitive skills in other contexts as well, specifically when subjects are required to choose the smaller of two food quantities to receive the larger in the "reverse contingency task" without extensive training (i.e., Boysen \& Berntson, 1995; Kralik, Hauser, \& Zimlicki, 2002).

We must also consider the possibility that the stress of separation influenced the behavior of the tamarins in this experiment. In each session, the recipient animal was confined to either the recipient cage within the home cage or inside an auxiliary cage outside the home cage. Although training measures were taken to habituate the animals to separation, some individuals continued to display anxiety-like behavior. Recipients inside the recipient cage sometimes exhibited piloerection and a preoccupation with exiting the recipient cage, ignoring the trays, and refusing food once it had been donated. The anxious behavior of the recipients may have affected the donors' choices. Furthermore, during control sessions when the recipient was fully removed from the cage, donors would sometimes invest little attention in the task and attempt to regain contact with their mate.

\section{Experiment 2: Low-Cost Donation}

In Experiment 2, we eliminated reward availability to the donor to minimize the possibility of the donors' behavior being affected

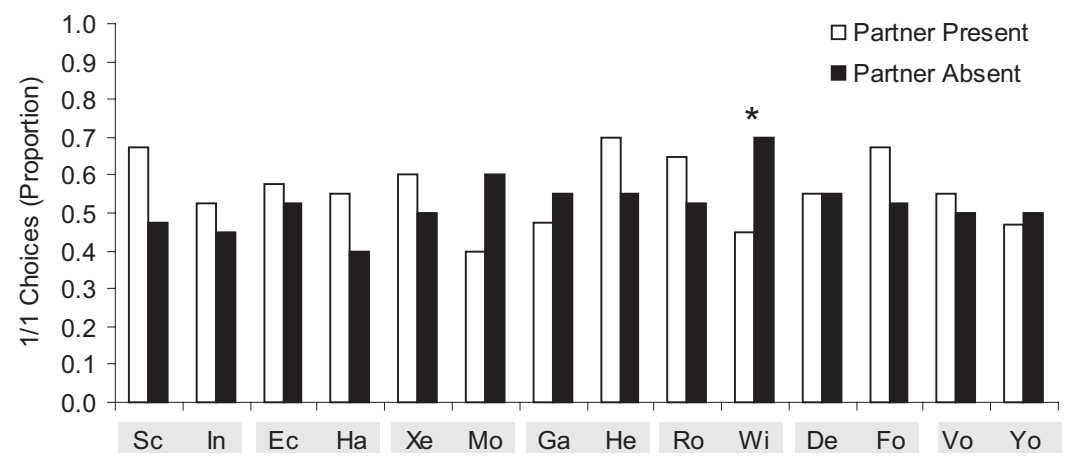

Figure 2. Proportion of $1 / 1$ (one reward to the donor and one reward to the recipient) choices made by each tamarin during the mate-present (open bars) and mate-absent (closed bars) conditions. Across the $y$-axis, mated pairs are shaded, with the female listed first and the male listed second, with the exception of the females Vo and Yo, whose mates were not tested. The difference was nonsignificant for 13 of 14 individuals. One tamarin chose the $1 / 1$ option significantly more often when the mate was absent than when the mate was present (Wi, two-tailed Fisher's exact test, $p=.041)$. ${ }^{*} p \leq .05$. 
by preoccupation with food. Tamarins were presented with the choice between two trays, one that delivered a single reward to their mate (the " $0 / 1$ " option) and one that did not provide rewards to either individual (the " $0 / 0$ " option). The distribution of benefits in Experiment 2 still allowed direct interspecific comparisons of donation behavior as it has been recently used by Burkart et al. (2007) with common marmosets and Vonk et al. (2008) with chimpanzees. A similar reward scenario was also used with chimpanzees in Jensen et al. (2006, Experiment 2). We also minimized separation distress for the tamarins by shortening the length of sessions and adjusting the experimental setup so mates were never fully isolated from one another. Finally, we included detailed measurements of recipient communication and motivation to allow us to determine whether the behavior of the recipient affected the likelihood of donation by the mate.

\section{Method}

\section{Subjects, Apparatus, Training Procedure}

The tamarins of Experiment 2 were the same as Experiment 1. An average interval of 3.5 months elapsed between Experiments 1 and 2 . The same donation apparatus and recipient cage were used, as well as a second cage identical to the recipient cage. No additional training or testing took place between Experiment 1 and Experiment 2.

\section{Testing Procedure}

Pairs were tested in the same order as they had been for Experiment 1 to equalize the interval between experiments. Each donor participated in four sessions consisting of 10 trials each, once daily for 4 consecutive days. All sessions took place between 9:30 and 11:30 a.m. Sessions alternated between mate-present and mate-absent (control) conditions, and the first condition was counterbalanced across individuals. The individual within each pair that had been randomly selected as the first recipient in Experiment 1 was the first donor of that pair in Experiment 2. Once one donor completed four sessions, the roles were reversed and the former recipient served as donor for the next four sessions (except for the two subjects whose mates did not meet the training criteria for Experiment 1). Two to 3 days elapsed between role reversals.

During the mate-present condition, the donor ranged in the home cage and the recipient was restricted to the recipient cage hanging in the front of the cage as in Experiment 1. An additional, empty auxiliary cage hung at the rear of the cage, which was closed.

During the mate-absent (control) condition, the donor ranged in the home cage and the mate was restricted to the auxiliary cage that hung in the rear of the home cage (rear auxiliary cage). The other recipient cage was in the same location as the mate-present condition, empty and secured shut (see Figure 3). This reflects a modification from Experiment 1 in which the mate was completely removed from the home cage during the mate-absent condition, and was intended to minimize separation distress. Individuals were now separated from the recipient by only the auxiliary cage mesh (see Figure 3).

All sessions were videotaped. On each trial, a single reward was placed on either the top or bottom tray so that one tray delivered

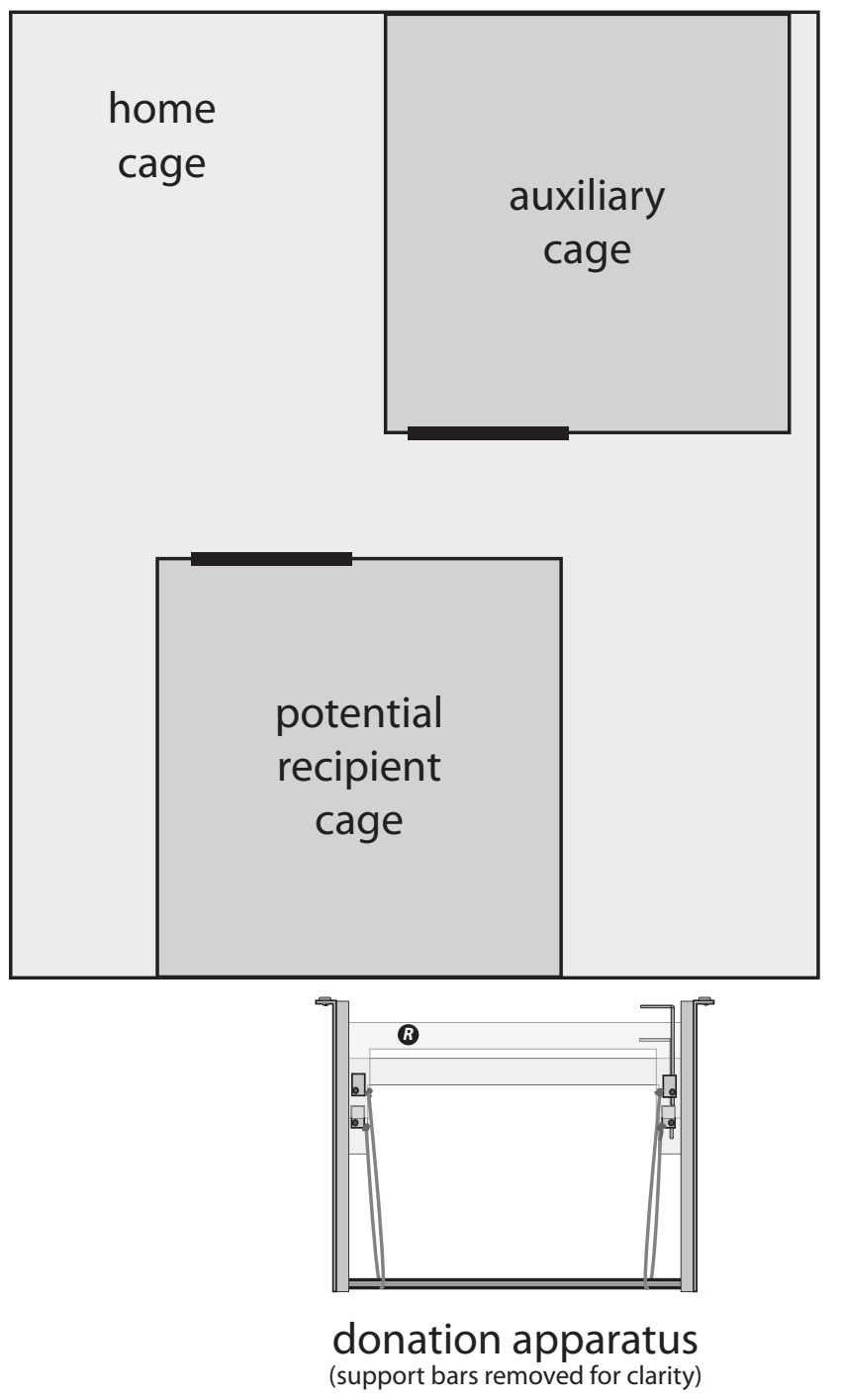

Figure 3. Top-down view of setup for Experiment 2. During matepresent conditions, the donor ranged in the home cage and had access to the handles on the donation apparatus, and the potential recipient was restricted to the recipient cage in the front of the home cage. During mate-absent (control) conditions, the donor ranged in the home cage and the mate was restricted to the rear auxiliary cage. Rewards are represented as in Figure 1. In this example, the top tray would be the " $0 / 1$ " tray (provides no reward to the donor and one reward to the recipient) and the bottom tray would be the " $0 / 0$ " tray (does not provide a reward to either animal).

a reward to the recipient (or empty recipient cage in the partnerabsent condition, tray " $0 / 1$ ") and the other tray was empty (tray "0/0"; see Figure 3). The donor could not obtain any rewards for pulling the trays.

The trays were pushed into reach of the donor once the experimenter (KAC) observed that the donor oriented its head toward the reward; pushing the trays into reach marked the start of the trial. The maximum trial length was $30 \mathrm{~s}$. The donor could make one of three responses: (a) pull the baited tray, (b) pull the unbaited tray, or (c) pull neither tray. The location of the reward was randomized between the top and bottom loca- 
tions, and the randomization was restricted so that within each session the reward was on the top tray on five trials and on the bottom tray on five trials. All rewards were of equal size, and only one reward type was used per pair (cookie, raisin, tuna, peanut, or burger, determined during preference tests prior to the onset of testing).

The tamarin's tray choice was scored by KAC during testing and independently by a second observer while coding the data from video. When food chirps were emitted by tamarins, KAC and a second experimenter (KKS) vocally noted the identity of the caller to enable later verification from videotape.

\section{Data Analysis}

As in Experiment 1, the data were analyzed at both the group and individual level using SPSS version 12.0 and VassarStats. Results were considered significant at $p \leq .05$.

The donor's response $(0 / 0,0 / 1$, or no response) was coded from video. From video we also coded the following behaviors of the recipient to evaluate the effect of the motivational or communicative state of the recipient on the donor's response: reaching toward the reward prior to the donor's response (a reach was defined as the extension of the recipients arm through the cage mesh toward the tray with fingers outstretched) and food chirping prior to the donor's response. Twenty percent of sessions were randomly selected and coded by a second observer. Rater agreement for tray choice was perfect, yielding Cohen's kappa of 1.0. Rater agreements for whether a food chirp or reach occurred were 0.747 and 0.882 , respectively.

Group analyses. To determine whether tamarins were more likely to choose the $0 / 1$ tray when their mate was present compared with when their mate was absent, we performed a binomial regression. Response (donation: $0 / 1$ or no donation: $0 / 0$ and no response) was the dependent variable, and condition (present or absent), block (first or second round of conditions), and position ( $0 / 1$ tray on top or bottom) were covariates. We combined the $0 / 0$ response with trials on which the tamarins did not respond because we were primarily interested in whether the proportion of $0 / 1$ responses changed across conditions. In addition, we performed a multinomial regression with all three potential response types $(0 / 0$, $0 / 1$, no response) using the same covariates as the binomial regression. Because the data included repeated measures from the same individuals, we included individual identity as a categorical variable to control for individual differences and remove the source of nonindependence.

To determine whether tamarins were more likely to donate when the recipient demonstrated interest in the food reward, we separately compared trials on which food chirping did and did not occur prior to the donor's choice and trials on which the recipient did and did not reach toward the food reward prior to the donor's choice, using paired Student's $t$ tests.

Individual analyses. To determine whether any individual tamarins were more likely to choose the $0 / 1$ tray when their partner was present compared with when the partner was absent, we performed a two-tailed Fisher's exact test on the data for each subject, collapsing across blocks.

\section{Results}

\section{Group Results}

Tamarins chose the $0 / 1$ tray an average of $33.0 \%(95 \% \mathrm{CI}=$ $19.9,46.1$ ) of trials during the partner-present condition and $30.0 \%$ $(95 \% \mathrm{CI}=21.5,38.5)$ of trials during the partner-absent condition. The binomial logistic regression failed to support our hypothesis that tamarins would donate more often when a recipient was present than when a recipient was not present, as there was no significant effect of condition ( $\mathrm{OR}=0.982, p=.929)$. There was no significant effect of tray position $(\mathrm{OR}=0.865, p=.473)$; that is, the tamarins did not significantly prefer the top or bottom tray regardless of reward location. The model did reveal a significant effect of block (OR $=1.653, p=.015)$, and indicated that tamarins chose the $0 / 1$ option significantly less in the second block of sessions than in the first block of sessions. The multinomial regression, which maintained a lack of response and the $0 / 0$ choice as separate response types, also resulted in a significant effect of block only $(p=.001)$. The decrease in donation in the second block of trials was primarily due to an increase in the number of trials on which the donor did not make any response (percentage no response: Block 1 partner present: $46.4 \%, 95 \% \mathrm{CI}=26.0,66.9$; Block 2 partner present: $61.4 \%, 95 \% \mathrm{CI}=40.7,82.2$; Block 1 partner absent: $42.9 \%, 95 \% \mathrm{CI}=6.0,59.7$; Block 2 partner absent: $66.4 \%, 95 \% \mathrm{CI}=48.8,84.0)$. There was no sex difference observed (two-tailed independent samples $t$ test on difference scores between proportion of $0 / 1$ choices present vs. absent), $t(12)=0.499, p=.627, d=0.266$.

We hypothesized that donors would be more likely to chose the 0/1 option when recipients were displaying an interest in the food reward. Cottontop tamarin food chirps are a reliable measure of motivational interest in food (Elowson, Tannenbaum, \& Snowdon, 1991). We retrospectively divided trials into those in which the recipient emitted at least one food chirp toward the reward prior to the donor making a response and trials during which no food chirps were emitted, and calculated each donor's proportion of donation responses on each trial type. One donor (Ro) was paired with a recipient that did not emit any food chirps (Wi) and was therefore excluded from this analysis. We performed a paired $t$ test to determine whether donation differed depending on recipient vocalizations. Tamarins donated less often when the recipient emitted food chirps; however, this difference narrowly failed to reach significance, $t(12)=2.147, p=.053, d=0.669$ (see Figure $4 \mathrm{~A})$. The donor that was excluded from the analysis because his partner never food chirped chose the $0 / 1$ option on $65 \%$ of trials in the partner-present condition and $10 \%$ of trials in the partnerabsent condition.

We also hypothesized that donors would be responsive to the actions of their mates and be more likely to chose the $0 / 1$ option when recipients reached toward the out-of-reach reward. As was done with analyses of food chirps, we divided trials into those in which the recipient exhibited at least one reach toward the reward prior to the donor making a response and trials during which the recipient did not reach for the reward. We performed a paired $t$ test to determine whether donation tendency differed depending on recipient gestures. Three donors (Mo, Ro, and Fo) were paired with recipients that never reached toward the food reward $(\mathrm{Xe}, \mathrm{Wi}$, and De, respectively) and were excluded from the analysis. Results indicated that tamarins donated significantly less often when the 


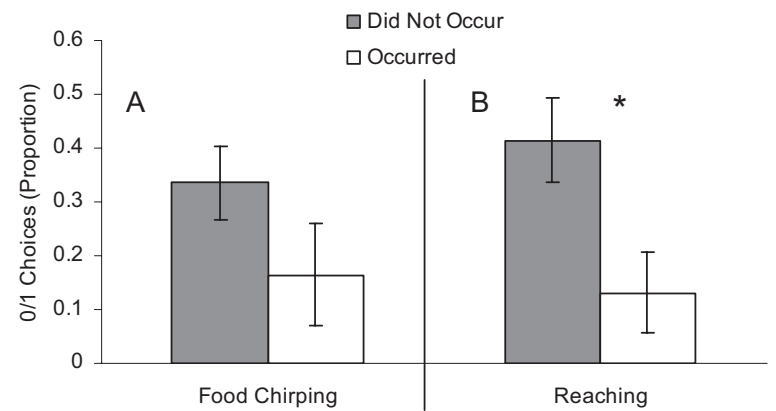

Figure 4. A. Mean proportion of 0/1 (no reward to the donor and one reward to the recipient) choices made on trials during which the recipient food chirped at least once (dark gray bars) compared with trials during which no food chirps were emitted by the recipient (light gray bars). For example, the left-most bar represents the mean proportion of 0/1 choices made on trials during which the recipient did not emit a food chirp. The difference just failed to reach significance, $t(12)=2.147, p=.053$. B. Mean proportion of $0 / 1$ choices made on trials during which the recipient reached toward the food reward at least once (dark gray bars) compared with trials on which the recipient did not reach toward the food reward (light gray bars). The difference was significant, $t(10)=6.340, p<.001$. $* p \leq .05$.

recipient reached toward the reward prior to the donor's response, $t(10)=6.340, p=.001, d=1.162$ (see Figure 4B). The donors that were excluded from the analysis because their partners never reached for the food reward performed as follows: Mo chose the $0 / 1$ option on $35 \%$ of partner-present trials and $30 \%$ of partnerabsent trials; Ro chose the $0 / 1$ option on $65 \%$ of partner-present trials and $10 \%$ of partner-absent trials; Fo chose the $0 / 1$ option on $35 \%$ of partner-present trials and $30 \%$ of partner-absent trials.

\section{Individual Results}

Three subjects (Ha, Ro, and Wi) completed only one partnerabsent condition because of refusal of the mate to enter the rear auxiliary cage. The results were nonsignificant for 12 of 14 tama- rins (all $p \mathrm{~s} \geq .18$ ). The two-tailed Fisher's exact test resulted in significance for 2 tamarins (female Xe: $p=.020$; male Ro: $p=$ .007). Ro donated significantly more often when his mate was present than absent; however, Xe chose the 0/1 tray more often when her mate was absent than when her mate was present (see Figure 5).

We performed an additional analysis to determine whether tamarins were more likely to donate if they had already been in the role of recipient. This may have occurred either because of reciprocity effects or a better appreciation of the task by individuals who had previously been recipients. However, there was no significant difference in donation between tamarins that were donors first and tamarins that were recipients first (independent samples $t$ test), $t(10)=0.736, p=.478, d=0.425$.

\section{Discussion}

In Experiment 2, the methodology was altered so that food preoccupation by the donor was reduced and separation distress for both the donor and recipient was minimized. However, as in Experiment 1, donors did not regularly provide rewards to recipients, and donors provided fewer rewards as the experiment progressed. Two individuals differed significantly from chance in the amount of $0 / 1$ choices made when a partner was present compared with absent. However, these findings were in opposite directions (one donated significantly more to a partner than to an empty cage, the other donated significantly less to a partner than to an empty cage) and, given the numerous statistical comparisons made in the individual analyses, it is most parsimonious to assume these significant findings arose by chance. It is surprising that we found that increases in displays of interest in the rewards by the potential recipients (food chirping and reaching toward the reward) were associated with decreases in donations.

\section{General Discussion}

We hypothesized that cottontop tamarins would act in the best interest of their long-term mates in a food donation task. This

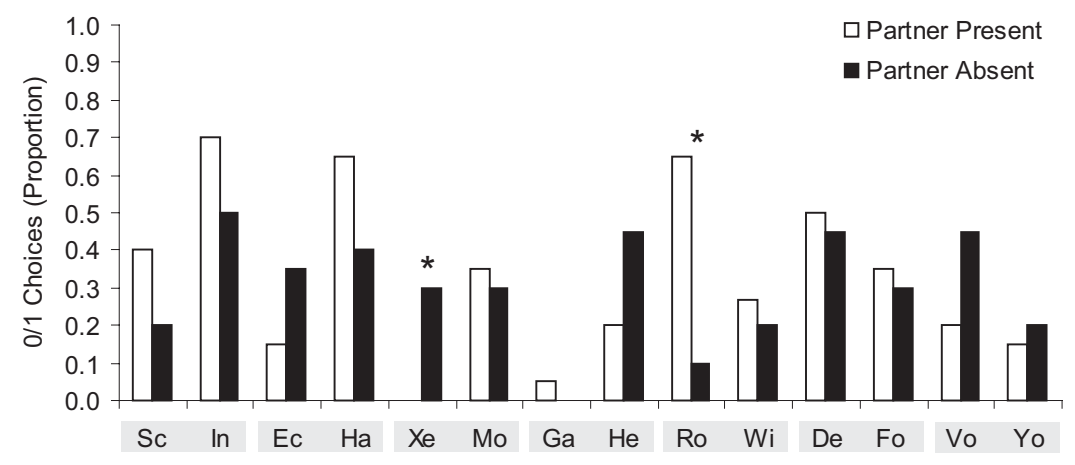

Figure 5. Proportion of 0/1 (no reward to the donor and one reward to the recipient) choices made by each tamarin during the mate-present (open bars) and mate-absent (closed bars) conditions. Across the $y$-axis, mated pairs are shaded, with the female listed first and the male listed second, with the exception of the females Vo and Yo, whose mates were not tested. The difference was nonsignificant for 12 of 14 individuals. One individual chose the 0/1 option significantly more often when the mate was present than when the mate was absent (Ro, two-tailed Fisher's exact test, $p=.007)$. One tamarin chose the $0 / 1$ option significantly more often when the mate was absent than when the mate was present (Xe, two-tailed Fisher's exact test, $p=.020$ ). ${ }^{*} p \leq .05$. 
hypothesis was generated on the basis of the cooperative social system of Callithrichids, which involves collaboration among many group members to coordinate offspring care, group foraging, and defense. The high attentiveness to social cues and extreme tolerance within Callithrichid social groups has led to speculation regarding cognitive adaptations for cooperative living (Burkart et al., 2007; Coussi-Korbel \& Fragaszy, 1995; Snowdon, 2001; Snowdon \& Cronin, 2007; van Schaik \& Kappeler, 2006). The performance of Callithrichids on social learning and cooperative tasks has supported the notion that the Callithrichids are attentive to group mates, able to temporally coordinate their actions to cooperate, and willing to provide food to conspecifics in some situations (Bugnyar \& Huber, 1997; Burkart et al., 2007; Cronin et al., 2005; Cronin \& Snowdon, 2008; Hauser et al., 2003;Humle \& Snowdon, 2008; Moscovice \& Snowdon, 2006; Werdenich \& Huber, 2002).

In the first experiment, cottontop tamarins were given the opportunity to provide food to their long-term mates at no additional cost to themselves. Donors could have provided rewards to their mates as a byproduct of a selfish act to obtain a reward for themselves. However, tamarins did not choose the tray that would provide rewards to their mates more often when the mate was present than when the mate was absent, failing to support the hypothesis that tamarins would act in the best interest of their mates. These findings are similar to those reported for chimpanzees (Jensen et al., 2006; Silk et al., 2005; Vonk et al., 2008).

We identified factors that may have inhibited donation in Experiment 1. The equality of tray options from the donor's perspective (they received a single reward regardless of tray choice) may have led tamarins to choose randomly. During all training prior to the experiment, only one reward was available on each trial; therefore, the tamarins may not have attended to the reward location in front of the mate once they identified rewards in front of themselves. Preoccupation with obtaining rewards has been suggested as an inhibitor of prosocial behavior in chimpanzees (Warneken \& Tomasello, 2006; Warneken et al., 2007). Also, some tamarins exhibited distress during the brief separation required in the control conditions, which may have affected their behavior at the apparatus. The second experiment minimized these factors.

The second experiment was also desirable because it presented tamarins with the reward locations that they had become familiar with during training sessions. During training, we had never exposed the tamarins to the exact reward scenario that they encountered during testing in Experiment 1. Namely, they never demonstrated an ability to distinguish between a tray with two rewards and a tray with a single reward. We had avoided presenting a double-baited tray because we were concerned that the tamarins would develop a logical preference for the double-baited tray and carry that preference over to testing sessions, resulting in a ceiling effect and minimizing our ability to detect differences between partner-present and partner-absent conditions. However, in the second experiment, only one reward was present on the trays. The tamarins had demonstrated an ability to discriminate between unrewarded and rewarded trays with a single reward during training.

Yet, in Experiment 2, when food preoccupation and the extent and duration of separation were minimized and familiar reward distributions were used, tamarins again did not show a significant donation preference. In Experiment 2, tamarins could choose between a tray that provided a reward for their mate and a tray that had no rewards. Unlike the first experiment in which donors responded on every trial, in Experiment 2 the tamarins often made no response. Tamarins made fewer pulls in the second block of trials, regardless of whether the partner was present or absent, indicating that their motivation to select a tray decreased as they were consistently unrewarded for their efforts.

We also tested the hypothesis that tamarins would provide rewards to recipients that displayed an interest in the reward. Donors may be unlikely to deliver rewards to recipients that were preoccupied with exiting the recipient cage and appeared uninterested in the food rewards. Chimpanzees may not provide help to conspecifics if the second individual does nothing to indicate that they are in need of assistance (Warneken et al., 2007). It is surprising that we found that tamarins delivered rewards significantly less often if the recipient made even a single reach toward the reward. These results are especially intriguing given the recent findings that common marmosets, another cooperatively breeding Callithrichid species, donated food to conspecifics in a nearly identical experimental design, and the likelihood of donation by common marmosets was unaffected by communication by the recipient (Burkart et al., 2007).

We hypothesized that cottontop tamarins would provide rewards to conspecifics in this study, in part due to previous studies of cooperative behavior in this species. We previously reported that pair-bonded cottontop tamarins coordinated actions in time and space to solve a cooperative task for food rewards and understood the role of the mate in the cooperative act (Cronin et al., 2005). Furthermore, we have reported continued cooperation when tamarins transition from obtaining equal rewards to being alternatively rewarded, then to having to compete for a single reward, and finally to continually having one cooperative partner rewarded while the other remains unrewarded (Cronin \& Snowdon, 2008).

Of most relevance to the current study is the final experiment reported in Cronin and Snowdon (2008). Cottontop tamarins were required to simultaneously extend handles to release a single, monopolizable reward to one actor, and the identity of the rewarded actor remained unchanged. Rates of cooperation were lower under this reward scenario than in the others, but cooperation was not extinguished, and tamarins completed approximately half of the trials. Given the lack of prosocial preferences in the current experiments, the continued cooperation on the part of the unrewarded actor in the previous study is of interest. The tamarins' previous experience in the cooperative problem-solving task may have increased their willingness to continue cooperating, either because they had a strong positive association with the task (having been previously rewarded on at least an average of half the trials in each previous condition) or because they continued sampling the task to determine whether the reward scheme had changed to again be more favorable. Our sample was too small to allow randomization of order of exposure to the conditions, so we cannot determine whether the rate of cooperation would have been less had the subjects not previously experienced more favorable reward scenarios. Although tamarins acted cooperatively in each condition, they also showed selfish motivation throughout the study through higher rates of pulling to obtain rewards for the self rather than the partner, shorter latency to pull to obtain rewards for the self, and an attempt to obtain the reward on nearly every trial 
in a condition in which a single, monopolizable reward was delivered equidistant from both subjects.

Another possible reason for the discrepancy between the cooperative behavior resulting in reward delivery in Cronin and Snowdon (2008) and the lack of reward delivery in the current study may be due to the lack of behavioral coordination required in the current study. Acting in concert with another individual, as was required in Cronin and Snowdon (2008), may be inherently rewarding (Schuster, 2002; Schuster \& Perelberg, 2004). In the current study, only the donor exerted effort, whereas the recipient was uninvolved in procuring the rewards. The notion that coordinated action by both individuals would have provided intrinsic reward and affected the behavior of the donor requires more thorough investigation.

Hauser et al. (2003) reported results that appear inconsistent with those reported here. They demonstrated that cottontop tamarins would pull food into reach of conspecifics that were trained to pull food into reach for them. The cottontop tamarins distinguished between trained altruists and trained defectors, and pulled more food into the reach of the former. Hauser et al. examined whether cottontop tamarins would reciprocate with individuals who either recently (within seconds) rewarded them or failed to provide them with a reward. In contrast, the current study examined whether cottontop tamarins would provide rewards to a conspecific who has neither provided nor denied them rewards immediately previously. The delivery of rewards to the trained altruist in Hauser et al. may have been facilitated by short-term reciprocity between tamarins that was not possible in the current study. Other differences between Hauser et al. and the current study prohibit direct comparison between results, most notably the lack of multiple tray choices in Hauser et al.

We hypothesized, as did Burkart and colleagues (2007), that cooperatively breeding primates would show prosocial tendencies not exhibited by chimpanzees in food donation tasks (Jensen et al., 2006; Silk et al., 2005; Vonk et al., 2008). Both the present study with cottontop tamarins and the Burkart et al. study with common marmosets were modeled closely after Silk et al. (2005), resulting in very similar experimental designs. However, common marmosets donated food to conspecifics, whereas cottontop tamarins did not. Could minor methodological differences between Burkart et al. and our study have led to the interspecific differences observed, or are there differences between cooperatively breeding common marmosets and cottontop tamarins that might explain their different performance?

The training stages of Burkart et al. (2007) and the present study were very similar. During test sessions, common marmosets were exposed to a few interspersed motivational trials on which the donors could pull a tray to provide themselves with a reward, which the tamarins were not. The common marmosets chose the $0 / 1$ tray significantly more often when a partner was present than when a partner was absent, even though motivational trials were interspersed in both conditions. Therefore, this methodological difference is unlikely to have contributed to the prosocial effect observed in Burkart et al. However, had we included motivational trials in the present study, the response rate of donor tamarins in Experiment 2 may have been greater, and it is conceivable that there could have been a greater rise in prosocial than nonprosocial choices.
A second difference is that the cottontop tamarins had prior experience with the donation experiment (Experiment 1) with a more favorable reward distribution from the donor's perspective. It is possible that the tamarins' previous experience with a reward scenario in which the donors could obtain rewards for themselves affected their willingness to donate to the mate when they could not obtain rewards in Experiment 2. They may have perceived the options as less optimal than they had previously experienced and therefore were less likely to provision their mates. This possibility is supported by observations that some primate species evaluate the relative value of benefits on the basis of previous experiences (i.e., Chen, Lakshminarayanan, \& Santos, 2006).

The smaller size of the testing cages $(30 \times 40 \times 53 \mathrm{~cm}$; length $\times$ width $\times$ height) used in Burkart et al. (2007) may also have contributed to the prosocial results observed in marmosets. The tamarin donors ranged home cages that had 50 times more space than the cages in which the marmoset donors were tested, after accounting for the space taken up by the recipient cage and auxiliary cage. Unlike the tamarins, the marmosets were removed from their home cage and the donors were temporarily contained in a small space where they experienced fewer alternative behavioral options than the tamarins. Differential results due to cage sizes have been reported in previous studies examining perspective taking and competition in chimpanzees (i.e., Bräuer, Call, \& Tomasello, 2007) and the potential impact of cage size should be considered in future comparative studies.

Finally, the social conditions from which the marmoset and tamarins donors were selected differed between the two studies. All marmoset subjects were regularly housed in groups with varying numbers of helpers present, whereas all tamarins tested were housed in monogamous pairs without helpers. The varied social composition experienced by the marmosets may have fostered more food transfers in nonexperimental contexts, which was reflected in their experimental results.

The possibility exists that methodological differences do not fully account for the different performance of common marmosets and cottontop tamarins. Common marmosets and cottontop tamarins may have experienced sufficiently different social or ecological pressures that have led them to express different prosocial tendencies. If evidence continues to accumulate indicating that capuchin monkeys and common marmosets demonstrate prosocial preferences and cottontop tamarins and chimpanzees do not, a finer examination of social and ecological factors shared by capuchins and common marmosets but not by cottontop tamarins and chimpanzees will be useful.

Further study is needed to answer the questions that emerge from these findings. All cooperatively breeding primates do not perform similarly on prosocial food donation tasks, but the factors that contribute to the extremely different prosocial tendencies of cottontop tamarins and common marmosets in this experimental context are unclear. It seems likely that cooperative breeders would have evolved cognitive strengths and strategies that suit them well in the cooperative societies in which they live and that prosocial behavior may result from this, but additional factors must be considered. We are now in the desirable position of having data on multiple species (common marmosets, cottontop tamarins, capuchins, chimpanzees) tested in quite similar experimental paradigms, and should collect data on additional species with this 
paradigm to elucidate the multiple factors that contribute to the emergence of prosocial behavior among conspecifics.

\section{References}

Boesch, C. (2002). Cooperative hunting roles among Tai chimpanzees. Human Nature, 13, 27-46.

Boysen, S. T., \& Berntson, G. G. (1995). Responses to quantity: Perceptual versus cognitive mechanisms in chimpanzees (Pan troglodytes). Journal of Experimental Psychology: Animal Behavior Processes, 21, 82-86.

Bräuer, J., Call, J., \& Tomasello, M. (2007). Chimpanzees readily know what others can see in a competitive situation. Animal Cognition, 10, $439-448$

Bugnyar, T., \& Huber, L. (1997). Push or pull: An experimental study on imitation in marmosets. Animal Behaviour, 54, 817-831.

Burkart, J. M., Fehr, E., Efferson, C., \& van Schaik, C. P. (2007). Otherregarding preferences in a non-human primate: Common marmosets provision food altruistically. Proceedings of the National Academy of Sciences, USA, 104, 19762-19766.

Chen, M. K., Lakshminarayanan, V., \& Santos, L. R. (2006). How basic are behavioral biases? Evidence from capuchin monkey trading behavior. Journal of Political Economy, 114, 517-537.

Coussi-Korbel, S., \& Fragaszy, D. M. (1995). On the relation between social dynamics and social learning. Animal Behaviour, 50, 1441-1453.

Cronin, K. A., Kurian, A. V., \& Snowdon, C. T. (2005). Cooperative problem solving in a cooperatively breeding primate (Saguinus oedipus). Animal Behaviour, 69, 133-142.

Cronin, K. A., \& Snowdon, C. T. (2008). The effects of unequal reward distributions on cooperative performance by cottontop tamarins, Saguinus oedipus. Animal Behaviour, 75, 245-257.

de Waal, F. B. M., Leimgruber, K., \& Greenberg, A. R. (2008). Giving is self-rewarding for monkeys. Proceedings of the National Academy of Sciences, USA, 105, 13685-13689.

Elowson, A. M., Tannenbaum, P. L., \& Snowdon, C. T. (1991). Foodassociated calls correlated with food preferences in cotton-top tamarins. Animal Behaviour, 42, 913-937.

Feistner, A. T. C., \& McGrew, W. C. (1989). Food-sharing in primates: A critical review. In P. K. Seth \& S. Seth (Eds.), Perspectives in primate biology (Vol. 3, pp. 21-36). New Delhi: Today \& Tomorrow's Printers and Publishers.

Ginther, A. J., Ziegler, T. E., \& Snowdon, C. T. (2001). Reproductive biology of captive male cottontop tamarins as a function of social environment. Animal Behaviour, 61, 65-78.

Hare, B., Melis, A. P., Woods, V., Hastings, S., \& Wrangham, R. (2007). Tolerance allows bonobos to outperform chimpanzees on a cooperative task. Current Biology, 17, 619-623.

Hare, B., \& Tomasello, M. (2004). Chimpanzees are more skilful in competitive than in cooperative cognitive tasks. Animal Behaviour, 68, 571-581.

Hauser, M. D., Chen, K. M., Chen, F., \& Chuang, E. (2003). Give unto others: Genetically unrelated cotton-top tamarin monkeys preferentially give food to those who altruistically give food back. Proceedings of the Royal Society of London: Series B, 270, 2363-2370.

Hirata, S. (2007). Competitive and cooperative aspects of social intelligence in chimpanzees. Japanese Journal of Animal Psychology, 57, $29-40$.

Humle, T., \& Snowdon, C. T. (2008). Socially biased learning in the acquisition of a complex foraging task in juvenile cottontop tamarins, Saguinus oedipus. Animal Behaviour, 75, 267-277.

Jensen, K., Hare, B., Call, J., \& Tomasello, M. (2006). What's in it for me? Self-regard precludes altruism and spite in chimpanzees. Proceedings of the Royal Society of London: Series B, 273, 1013-1021.
Kralik, J., Hauser, M. D., \& Zimlicki, R. (2002). The relationship between problem solving and inhibitory control: Cotton-top tamarin (Saguinus oedipus) performance on a reversed contingency task. Journal of Comparative Psychology, 116, 39-50.

Lakshminarayanan, V. R., \& Santos, L. R. (2008). Capuchin monkeys are sensitive to others' welfare. Current Biology, 18, R999-R1000.

Lowry, R. (2008). VassarStats [Computer software]. Available from http:// faculty.vassar.edu/lowry/vassarstats.html

Moscovice, L. R., \& Snowdon, C. T. (2006). The role of social context and individual experience in novel task acquisition in cottontop tamarins, Saguinus oedipus. Animal Behaviour, 71, 933-943.

Muller, M. N., \& Mitani, J. C. (2005). Conflict and cooperation in wild chimpanzees. Advances in the Study of Behavior, 35, 275-331.

Nishida, T. (1983). Alpha status and agonistic alliance in wild chimpanzees. Primates, 24, 318-336.

Riss, D., \& Goodall, J. (1977). The recent rise to the alpha-rank in a population of free-living chimpanzees. Folia Primatologica, 27, 134 151.

Savage, A., Giraldo, L. H., Soto, L. H., \& Snowdon, C. T. (1996) Demography, group composition, and dispersal in wild cotton-top tamarin (Saguinus oedipus) groups. American Journal of Primatology, 38 , $85-100$.

Schuster, R. (2002). Cooperative coordination as a social behavior. Human Nature, 13, 47-83.

Schuster, R., \& Perelberg, A. (2004). Why cooperate? An economic perspective is not enough. Behavioral Processes, 66, 261-277.

Silk, J. B., Brosnan, S. F., Vonk, J., Henrich, J., Povinelli, D. J., Richardson, A. S., et al. (2005, October 27). Chimpanzees are indifferent to the welfare of unrelated group members. Nature, 437, 1357-1359.

Snowdon, C. T. (1996). Parental care in cooperatively breeding species. In J. S. Rosenblatt \& C. T. Snowdon (Eds.), Parental care: Evolution, mechanisms and adaptations (pp. 643-689). San Diego, CA: Academic Press.

Snowdon, C. T. (2001). Social processes in communication and cognition in Callitrichid monkeys: A review. Animal Cognition, 4, 247-257.

Snowdon, C. T., \& Cronin, K. A. (2007). Cooperative breeders do cooperate. Behavioural Processes, 76, 138-141.

van Schaik, C. P., \& Kappeler, P. M. (2006). Cooperation in primates and humans: Closing the gap. In P. M. Kappeler \& C. P. van Schaik (Eds.), Cooperation in primates and humans: Mechanisms and evolution (pp. 3-21). Berlin: Springer-Verlag.

Vonk, J., Brosnan, S. F., Silk, J. B., Henrich, J., Richardson, A. S., Lambeth, S. P., et al. (2008). Chimpanzees do not take advantage of very low cost opportunities to deliver food to unrelated group members. Animal Behaviour, 75, 1757-1770.

Warneken, F., Hare, B., Melis, A. P., Hanus, D., \& Tomasello, M. (2007). Spontaneous altruism by chimpanzees and young children. PLoS Biology, 5, 1-7.

Warneken, F., \& Tomasello, M. (2006, March 3). Altruistic helping in human infants and young chimpanzees. Science, 311, 1301-1303.

Watts, D. P. (1998). Coalitionary mate guarding by male chimpanzees at Ngogo, Kibale National Park, Uganda. Behavioral Ecology and Sociobiology, 44, 43-55.

Watts, D. P., \& Mitani, J. C. (2001). Boundary patrols and intergroup encounters in wild chimpanzees. Behaviour, 138, 299-327.

Werdenich, D., \& Huber, L. (2002). Social factors determine cooperation in marmosets. Animal Behaviour, 64, 771-781.

Received June 27, 2008

Revision received December 9, 2008 Accepted December 16, 2008 